\title{
Correspondence
}

\section{Gold exacerbates adjuvant arthritis in the rat}

SIR, The selection of drugs for clinical testing as 'slow acting' or 'disease modifying' agents in rheumatoid arthritis (RA) would be greatly facilitated if a suitable animal model was available as a screening test. Rat adjuvant arthritis has been tested in this regard and, despite some claims by others, ${ }^{1}$ we have in this laboratory consistently failed to detect any inhibition of disease by drugs such as gold and penicillamine. $^{23}$ These experiments have, however, involved administration of the drugs for only relatively short periods compared with the 3-6 months necessary to achieve a response in RA patients. For this reason, we have now studied the influence on adjuvant disease of 12 weeks' pretreatment with gold injections.

Sprague-Dawley rats, initially weighing approximately $140 \mathrm{~g}$, received twice weekly intramuscular injections of sodium aurothiomalate (Myocrisin) $5 \mathrm{mg}$ or $0.1 \mathrm{mg}$, or normal saline for 3 months. All were then challenged with an injection of $0.6 \mathrm{mg}$ dead tubercle bacilli in $0.1 \mathrm{ml}$ mineral oil into a hind footpad. The techniques used, including the method of scoring arthritis (by an observer unaware of

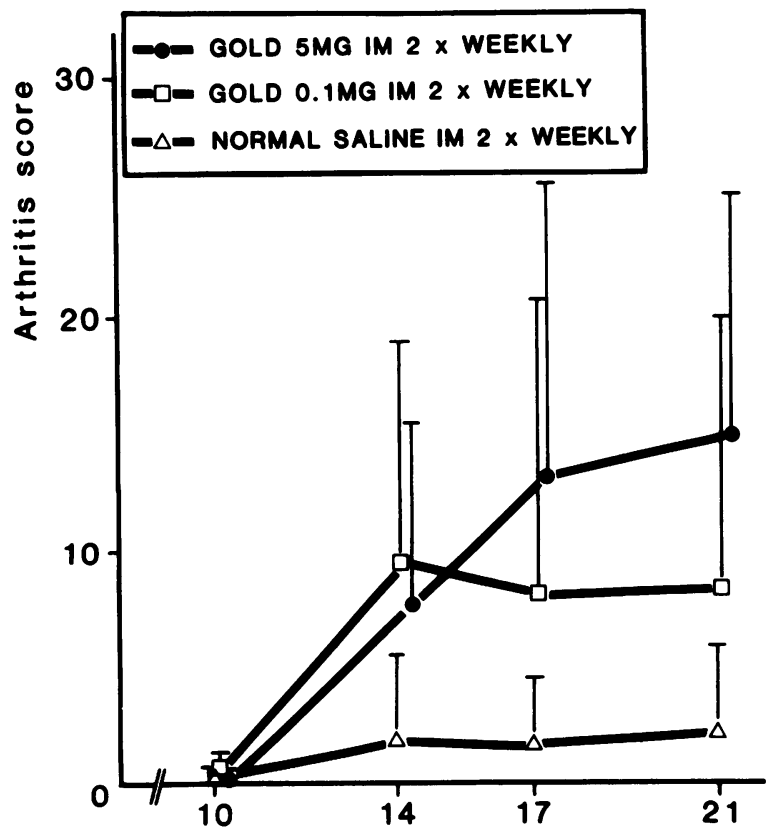

Days following adjuvant injection

Fig. 1 Mean joint scores after injection. treatment allocation), were the same as those employed previously. ${ }^{2}$ Intramuscular injections were continued during the period of observation.

Within 3 days of starting the intramuscular injections rats in the high-dose group became obviously toxic, lost weight rapidly, and $50 \%$ died. However, the remainder of the group gradually recovered despite continuing to receive gold injections, and by 2 weeks these animals were gaining weight at the same rate as those in the other groups. This curious pattern of toxicity and 'tolerance' remains unexplained. We have noted it before. ${ }^{2}$

At the end of the 3 months' pretreatment 7 rats in each group were challenged with adjuvant. At this stage there was no significant difference in weight between the 3 groups, and the mean weight was $464 \mathrm{~g}$ (SD 52-26). Fig. 1 shows the mean joint scores (+1 SD). Such older rats are less susceptible to adjuvant disease; hence the relatively low arthritis scores in the animals receiving saline. By contrast, those receiving the higher dose of gold developed significantly more severe arthritis (at day $21: p=0.011$, MannWhitney $U$ test). The animals receiving the lower dose of gold (comparable to the doses used in RA patients) developed arthritis of intermediate severity. However, with the small numbers involved the difference between their scores and those of the control group does not reach statistical significance.

The finding that gold injections exacerbate adjuvant arthritis is at first sight unexpected. However, these results confirm consistent past experience in this laboratory (using shorter courses of the drug), and we have no doubt that this is a genuine effect. Unexpected results can sometimes be more informative than an anticipated outcome.

Much discussion about the action of gold has in the past been based on experiments in which the drug has been added to in-vitro systems. It may be that effects observed in the intact animal are more significant. We speculate that this effect of the drug may be a pointer to its mechanism of action in RA. Experiments by Vernon-Roberts and his colleagues ${ }^{4}$ point to a suppression of macrophage function by gold. Gold could exert its influence in both situations by modifying phagocyte activity: in RA by interfering with phagocytosis of immune complexes within the joint and in adjuvant disease by preventing the clearance of disseminated mycobacterial deposits. In the rat it may be possible to test this.

Bone and Joint Research Unit,

D. O. HASKARD London Hospital Medical College, H. L. F. CURREY 25-29 Ashfield Street, London E1 2AD

\section{References}

1 Newbold B B. Chemotherapy of arthritis induced in rats by mycobacterial adjuvant. Br J Pharmacol 1963; 21: 127-36. 
2 Jessop J D, Currey H L F. Influence of gold salts on adjuvant arthritis in the rat. Ann Rheum Dis 1968; 27: 577-81.

3 Liyanage S P, Currey H L F. Failure of oral D-penicillamine to modify adjuvant arthritis or immune response in the rat. Ann Rheum Dis 1972; 31: 521 .

4 Jessop J D, Vernon-Roberts B, Harris J. Effects of gold salts and prednisolone on inflammatory cells. 1. Phagocytic activity of macrophages and polymorphs in inflammatory exudates studied by a 'skin-window' technique in rheumatoid and control patients. Ann Rheum Dis 1973; 32: 294-300.

\section{Rheumatoid arthritis and type 1 diabetes mellitus}

SIR, I read with interest the letter from Dr Rudge. ${ }^{1}$ We had reported a study ${ }^{2}$ in which we demonstrated an increased prevalence of type 1 diabetes and autoimmune thyroid disease in close relatives of patients with classical or definite rheumatoid arthritis. The rheumatoid patients demonstrated an increased prevalence of organ specific autoantibodies when compared to a control group with degenerative joint disease. We also included several pedigrees demonstrating the close relationship between rheumatoid arthritis and type 1 diabetes within some families. Dr Rudge's study was carried out at Nottingham along similar lines, but her study did not confirm our findings. It was mentioned by her that both the hospitals in which our study was carried out have an interest in the immunogenetics of these diseases and that this could attract the sort of cases we were interested in. In fact both rheumatology clinics service busy districts and there were very few referrals from outside our catchment areas. I suspect that this alone is not enough to explain the discrepancy.

Our original hypothesis was that the increased prevalence of type 1 diabetes in close relatives of rheumatoid patients could be predicted from the association both these diseases have with HLA DR4. We mentioned in the concluding paragraph of our paper that we would like to study HLA genotypes in families where one sibling had rheumatoid arthritis and another sibling had type 1 diabetes or other autoimmune endocrine disease. Since our paper was published we have studied some of the original families reported and others not included in the study; we have also studied some patients with coexistent type 1 diabetes and rheumatoid arthritis. Although this work is at a preliminary stage there is no clear association between these two diseases and HLA DR4 in the families and individual patients studied.

The pedigrees of families with coexistent rheumatoid arthritis and type 1 diabetes suggest a relationship between these two diseases, and we are confident that a mechanism for the common genetic susceptibility will be identified.

St Bartholomew's Hospital,

D. J. B. THOMAS

West Smithfield,

London EC1A 7BE

\section{References}

1 Rudge S. Rheumatoid arthritis and type 1 diabetes mellitus. Ann Rheum Dis 1983; 42: 603.
2 Thomas D J B, Young A, Gorsuch A N. Bottazzo G F, Cudworth A G. Evidence for an association between rheumatoid arthritis and autoimmune endocrine disease. Ann Rheum Dis 1983; 42: 297-300.

\section{Fatal bronchiolitis obliterans associated with chrysotherapy}

SIR, In the 'Discussion' section of their report of a case of fatal bronchiolitis obliterans (OB) associated with chrysotherapy Dr Holness and others ${ }^{1}$ state that a cystinuric patient given penicillamine succumbed to $O B$. The reference was to a letter of mine ${ }^{2}$ about penicillamine and $O B$. I had carried out a postal inquiry after deaths from $O B$ had been reported ${ }^{3}$ among patients with rheumatoid arthritis, some of whom had also been given penicillamine. In fact I failed to discover any further penicillamine-associated deaths from definite OB. One cystinuric patient had died from unspecified pulmonary disease which, in retrospect but without histological data, the reporting clinician thought might have been $\mathrm{OB}$. I categorised this diagnosis as 'uncertain'. To the best of my knowledge this extremely doubtful case represents the only published reference to $O B$ as a complication of cystinuria or its treatment. Dr Holness, by omitting my qualifying comment that the diagnosis of $O B$ in this instance was unlikely to have been correct quite unjustifiably made concrete what was insubstantial. The 'fact' that $O B$ has been associated with penicillamine treatment of cystinuria may now (and probably will) seep further into the literature and become part of received opinion. The 'fact', Sir, is no fact, and I write in the faint hope that its transformation into undying myth may be aborted.

7 Astley Close, W. H. LYLE

Leamington Spa CV32 6PT

\section{References}

1 Holness L, Tenenbaum J, Cooter N B E, Grossman R F. Fatal bronchiolitis obliterans associated with chrysotherapy. Ann Rheum Dis 1983; 42: 593-6.

2 Lyle W H. D-penicillamine and fatal obliterative bronchiolitis. $\mathrm{Br}$ Med J 1977; i: 105.

3 Geddes D M, Corrin B, Brewerton D A, Davies R J, TurnerWarwick M. Progressive airway obliteration in adults and its association with rheumatoid disease. $Q J$ Med $1977 ; 184: 427-44$.

\section{Salicylate-NSAID interactions}

Sir, We were interested in the paper of Garrett and coworkers ${ }^{1}$ concerning interactions between salicylates and other NSAIDs using a rat model of inflammation. The authors found a lack of additive effect or antagonism between salicylates and most of the other NSAIDs tested, although they did not show whether this was likely to represent a pharmacokinetic or pharmacodynamic interaction.

In the previous study in patients with rheumatoid arthritis quoted by Garrettet al. there was no additive effect between 